\title{
Ubiquitin-Conjugating Enzyme E2 R1
}

National Cancer Institute

\section{Source}

National Cancer Institute. Ubiquitin-Conjugating Enzyme E2 R1. NCI Thesaurus. Code C101303.

Ubiquitin-conjug ating enzyme E2 R1 (236 aa, 27 kDa) is encoded by the human CDC34 gene. This protein plays a role in the processing of ubiquitin for protein modification. 